\title{
The activity of the leaf essential oil of Philippine Piper betel against dermatophytes and Candida albicans
}

\author{
Delia De Castro-Ontengco ${ }^{1 *}$ and Teresita Capal ${ }^{2}$
}

\begin{abstract}
Superficial mycotic infections are treatable conditions and they rank as the third most common illness globally, next to headaches and tooth decays. Piper betel $L$. is an aromatic plant with heart-shaped leaves that play a role in traditional medicine. This study harnessed the antidermatophytic potential of the essential oil extracted from the leaves of Piper betel, PBLO, Philippine variety. Using serial twofold dilutions of PBLO, including selected PBLO constituents, to challenge Candida albicans ATCC 10231 and clinical isolates of filamentous dermatophytes, results indicate that the Minimum Inhibitory Concentration, MIC, of the essential oil extract was $625 \mathrm{ug} / \mathrm{ml}$ for C. albicans and MICs of $\leq 156$ $\mathrm{ug} / \mathrm{ml}$ for Microsporum canis, Microsporum gypseum, Trichophyton mentagrophytes, Trichophyton rubrum, and Trichophyton tonsurans. Data on known PBLO constituents suggest that eugenol plays a major role in PBLO's antidermatophytic activity, followed by chavicol. Chavicol and eugenol exhibited the same MIC, $625 \mathrm{ug} / \mathrm{ml}$, against $C$. albicans ATCC 10231. A eugenol MIC of $\leq 156 \mathrm{ug} / \mathrm{ml}$ was observed against M. canis, T. mentagrophytes, T. rubrum, and T. tonsurans; and $312.5 \mathrm{ug} / \mathrm{ml}$ against $M$. gypseum. Chavicol MICs of $312.5 \mathrm{ug} / \mathrm{ml}$ and $625 \mathrm{ug} / \mathrm{ml}$ were observed against Trichophyton spp. and Microsporum spp., respectively. Results of the study suggest the potential of $P$. betel essential oil as an alternative to conventional antifungals for the treatment of dermatophytosis. With the presence of many active constituents in PBLO essential oil, antifungal resistance is unlikely to happen, as multiple mutations will be required to overcome the mechanism/s of action of each of these constituents.
\end{abstract}

Keywords: Candida albicans, Microsporum spp, Trichophyton spp, eugenol, chavicol, Minimum Inhibitory Concentration, $P$. betel's antifungal activity

\section{Introduction}

Fungal skin infection may be caused by serious C. albicans and could affect over a million adults and children globally because the tools are not available for rapid diagnosis and treatment. Nearly a billion people have a fungal infection of the skin, the third most common illness internationally, after headaches and dental caries (GAFFI, 2017). Skin mycoses affect more than $20-25 \%$ of the world's population, making them the most frequent form of infection (Bassiri-Jahromi and Khaksari, 2009). WHO reports that in large geographical areas tinea capitis is present in up to $19.7 \%$ of the general population in developing countries (WHO, 2005).

Superficial mycotic infections are treatable conditions seen

\footnotetext{
${ }^{1}$ Graduate School, University of Santo Tomas

${ }^{2}$ Industrial Technology Development Institute - Materials Science

Division, Department of Science and Technology, Philippines

*Corresponding email: dcontengco@gmail.com

Date Submitted: 28 December 2018

Date Accepted: 11 August 2019
}

in daily clinical practice, and dermatophytes or ringworm infections are the main conditions. The Philippines has a warm and humid tropical climate with an average temperature of $27-$ $33^{\circ} \mathrm{C}$ and a relative humidity of $77-83 \%$, conditions contributing to a high prevalence of fungal infections. Local socio-economic conditions and cultural practices can also influence the incidence of dermatophyte infections in a given area. Handog and Dayrit (2005), in a four-year study, reported that dermatophytosis ranked second among the most common causes for consultation in local dermatology clinics. Dermatophytosis, also called tinea or ringworm, is a fungal disease of the outer layers of the skin, hair and nail caused by the following genera: Trichophyton, Microsporum and Epidermophyton, constitutively called dermatophytes. They cause a wide spectrum of diseases that range from guild scaling disorder to one that is generalized and highly inflammatory (Baron, 1996). Dermatophytes or keratinophilic fungi produce extracellular keratinases that can hydrolyze and utilize keratin as their source of nutrients (Reiss et al., 2018).

Aromatic plants and their essential oils have been used historically for their antimicrobial properties, as well as for 
cosmetic and other medicinal applications. In vitro screening programs, based on folkloric practices, have validated traditional uses and provided insights into active compounds (Caburian and Osi, 2010; Ontengco et al., 1999). Screening assays on plant essential oils have produced significant results as antibacterials and antifungals (Ali et al., 2010; Ontengco et al., 2004.; Moghimipour et al., 2009). Plant essential oils are considered to be bactericidal and fungicidal that are typically active at concentrations of $5 \%$ or less. Oils with large amounts of aldehydes, phenols or alcohols generally show the greatest antimicrobial activity. Microbicidal activity is directly related to the solubility of oil components in microbial membranes and disruption of associated functions (Thormar et al., 2010).

Piper betel L., locally known as "ikmo," is a climbing vine with aromatic heart-shaped leaves that play a role in traditional medicine. The leaf with coconut oil is warmed and applied on the abdomen to relieve flatulence or colic pain. Leaves, together with lime and betel nut, constitute the Filipino's masticatory. Its use is believed to help preserve the teeth. Fresh, crushed leaves are used as antiseptic for cuts and wounds. The chief constituent of the leaves is their volatile oil, betel oil, which contains two phenols: betelphenol (chavibetol) and chavicol. Other constituents are eugenol, allyl pyrocatechin, terpene, cineol, caryophyllene, cadinene, menthone. This investigation aimed to harness the antidermatophytic potential of the essential oil extracted from the leaves of Piper betel, Philippine variety.

\section{Materials and Methods}

\section{Piper betel leaves}

Three $\mathrm{kg}$ of fresh mature leaves of Piper betel were collected in May from Pugo, La Union. A complete portion of the plant was deposited, identified and authenticated by a curator of University of Santo Tomas, Research Center for the Natural Sciences. Dirt and topsoil were washed, cut into small pieces, and air-dried. The dry leaves were placed in an Erlenmeyer flask. Water was added to cover the leaves and boiling chips were included to prevent an increase of pressure inside the receiving vessel. A clavenger tube receiver was attached to a condenser and was fitted to the Erlenmeryer flask. Hydrodistillation or water distillation was carried out at low heat until no more oily drops was gathered from the clavenger tube. Oil was allowed to drip slowly into amber vials. Moisture was removed using anhydrous sodium sulfate, and stored at 2$8^{\circ} \mathrm{C}$ prior to its use (Ontengco et al. 1999).

\section{Controls}

Chavicol, cineole, and eugenol (all from Sigma-Aldrich) are known constituents of PBLO and were used as controls. PBLO extract and controls were filter-sterilized prior to use.

\section{Dermatophytes}

Candida albicans ATCC 10231 (Biological Sciences Department-United Laboratories Culture Collection) and preidentified clinical isolates $M$. canis, M. gypseum, $T$. mentagrophytes, T. rubrum, and T. tonsurans were provided by the Mycology Department of the University of the Philippines, College of Public Health, Manila. Candida albicans was grown in Sabouraud Dextrose Broth (SDB) at $28^{\circ} \mathrm{C}$ for $24-48 \mathrm{hrs}$ while the filamentous fungi were grown in $\mathrm{SDB}$ at $28^{\circ} \mathrm{C}$ for 5-7 days. The turbidity of all fungal suspensions was adjusted to a density equivalent to McFarland 0.5 or $\sim 10^{6} \mathrm{CFU} / \mathrm{ml}$ (Murray, 1999). The adjusted suspension was diluted 1:10 with SDB, giving a final inoculum density of $\sim 10^{5} \mathrm{CFU} / \mathrm{ml}$.

\section{Determination of minimum inhibitory concentration (MIC)}

The modified agar dilution method (Ontengco et al., 2004) was employed to determine the MICs of PBLO and the standard chemical constituents: chavicol, cineole, and eugenol against the dermatophytes and C. albicans. Briefly, PBLO or control constituent was emulsified in $20 \%$ Tween 80 and distilled water (0.2:0.12:1.68), serially diluted twofold with distilled water, and mixed with Sabouraud Dextrose Agar (SDA) to final oil or chemical constituent concentrations of $156 \mathrm{ug} / \mathrm{ml}$ to $5000 \mathrm{ug} / \mathrm{ml}$. The lowest concentration used was $156 \mathrm{ug} / \mathrm{ml}$ following the literature recommendation. The oil- or constituent-agar mixtures were allowed to solidify and the diluted fungal suspensions were spotted on pre-identified portions of the agar plates at a constant volume of $10 \mathrm{uL}(\sim 1000 \mathrm{CFU} / \mathrm{spot})$ and allowed to dry. The plates inoculated with $C$. albicans were incubated at $28^{\circ} \mathrm{C}$ for $48 \mathrm{hrs}$, while those with filamentous fungi were incubated at $28^{\circ} \mathrm{C}$ for 7 days, and further up to 21 days for a final negative or no-growth reading. Vehicle and untreated controls were included following the same procedure. The MIC was read as the least concentration of oil or constituent that failed to exhibit fungal growth after $4 \mathrm{~h}$ hrs for $C$. albicans and within 21 days for Microsporum spp, and Trichophyton spp. in relation to control plates.

\section{Results and Discussion}

Freshly extracted PBLO was a light yellow, aromatic liquid which turned pale brown on aging. The characteristic odor of the leaves and the oil is due to chavicol (Umali-Stuart, n.d.).

The Minimum Inhibitory Concentrations of the PBLO essential oil extract and the control constituents are exhibited in Table 1. 
Ontengco \& Capal: The activity of the leaf essential oil of Philippine Piper betel

Table 1. Minimum Inhibitory Concentrations of PBLO extract and controls vs dermatophytes and C. albicans

\begin{tabular}{l|l|l|l|c}
\hline \multirow{2}{*}{\multicolumn{1}{c}{ Fungal isolate }} & \multicolumn{4}{c}{ MIC (ug/ml) } \\
\cline { 2 - 5 } & $\begin{array}{c}\text { PBLO essential } \\
\text { Oil }\end{array}$ & chavicol & cineole & eugenol \\
\hline C. albicans ATCC 10231 & 625 & 625 & $>2500$ & 625 \\
M. canis & $\leq 156$ & 625 & $>2500$ & $\leq 156$ \\
M. gypseum & $\leq 156$ & 625 & $>2500$ & 312.5 \\
T. mentagrophytes & $\leq 156$ & 312.5 & $>2500$ & $\leq 156$ \\
T. rubrum & $\leq 156$ & 312.5 & $>2500$ & $\leq 156$ \\
T. tonsurans & $\leq 156$ & 312.5 & $>2500$ & $\leq 156$ \\
\hline
\end{tabular}

The above results indicate that PBLO is a potent antifungal against filamentous dermatophytes at $\leq 156 \mathrm{ug} / \mathrm{ml}$. and against C. albicans at $625 \mathrm{ug} / \mathrm{ml}$. Caburian and Osi (2010) stated that the leaf oil possesses significant antibacterial and antifungal activity against Staphylococcus aureus, Streptococcus pyogenes, C. albicans and T. mentagrophytes with MIC values of $125 \mu \mathrm{g} / \mathrm{ml} ; 15.60 \mu \mathrm{g} / \mathrm{ml} ; 250 \mu \mathrm{g} / \mathrm{ml}$ and $195 \mu \mathrm{g} / \mathrm{ml}$, respectively. Data produced by this study show lower MIC against $T$. mentagrophytes at $<156 \mathrm{ug} / \mathrm{ml}$, but higher MIC against C. albicans at $625 \mathrm{ug} / \mathrm{ml}$. Various factors may account for these differences, such as inoculum density in the susceptibility method used, sensitivity of the dermatophytes tested, or the constituent content and concentration in the betel oil used for the study. The latter may be due to time of the year or plant growth period the leaves were harvested, soil and environmental conditions among others.

Data on the control chemical constituents tested suggest that eugenol is largely responsible for PBLO's antidermatophytic activity, followed by chavicol. Cineole exhibited quite a high MIC for all dermatophytes tested and it may be presumed that it does not play a role in betel oil's antifungal activity.

Caburian and Osi (2010) identified the chemical components of the oil via gas chromatography-mass spectroscopy as 5-(2-propenyl)-1, 3-benzodioxole, eugenol isomer and caryophyllene among others. Rimando et al. (1986) reported that the chief constituent of the leaves is the volatile oil, which they called Betel oil. Further, they indicated the following as the major chemical constituents of the betel oil: two phenols, betelphenol (chavibetol) and chavicol. The volatile oil has $0.8-1.8 \%$ of chavicol, betelphenol, eugenol, allyl pyrocatechin, terpene, cineol, caryophyllene, cadinene, menthone.

These different constituents have major and/or minor roles in antisepsis. They may also act together in synergy or some may be antagonistic or additive.

Eugenol has a spicy, clove-like aroma. It is a phenol and is considered bacteriostatic against both fungi (Duke, 1985) and bacteria (Thomson, 1978). Contrary to the results of the present study - wherein eugenol exhibited an MIC of $\leq 156 \mathrm{ug} / \mathrm{ml}$ for $T$. rubrum, Duke's study reported eugenol as inhibitory at $256 \mu \mathrm{g} /$ $\mathrm{ml}$ to $7 / 14(50 \%)$ of T. rubrum strains. Mycelial growth and conidia germination were likewise inhibited, causing abnormalities in the morphology of the dermatophyte and exhibiting wide, short, twisted hyphae and decreased conidiogenesis. It was further suggested that eugenol exerts antifungal effects on the cell wall and cell membrane of $T$. rubrum by a mechanism that seems to involve the inhibition of ergosterol biosynthesis. Ergosterol is an important structural component of fungal cell membranes, maintaining membrane fluidity and a permeability barrier, and is essential for fungal cell viability. Its depletion interfere with the "bulk" functions of ergosterol in fungal cell membranes, leading to alterations in membrane fluidity and activities of several membrane bound enzymes (e.g., chitin synthase) (Maetens, 2004), which may induce cell membrane damage and morphological changes. The lower ergosterol content interferes with the integrity and functionality of the cell membrane. Eugenol can be hepatoxic at higher doses (de Oliveira Pereira, 2012). In a separate study, Park and co-workers (2007) analyzed the mechanism of action of eugenol. Modifications in T. mentagrophytes hyphal ultrastructure were observed, such as destruction of the inner mitochondrial membranes and cell wall, as well as expansion of endoplasmic reticulum near the cell membranes.

Chavicol is a natural phenylpropene, a type of organic compound. It is considered an antiseptic, twice as strong and isometric with eugenol (Rimando et al., 1986; Umali-Stuart, n.d.). It is a colorless liquid found together with terpenes in betel oil. It is miscible with alcohol, ether, and chloroform. Chavicol is used as an odorant in perfumery (Atkinson, 2018). 
Pure cineol is a colorless, optically inactive liquid, the odor of which resembles that of camphor and which crystallizes in the cold. It also possesses antiseptic properties, but as indicated by this study's data, it is not antidermatophytic.

\section{Conclusions and Recommendations}

Results of the present study indicate the antidermatophytic potential of $P$. betel essential oil as a raw material for topical applications against dermatophytes and C. albicans.

Future directions may include expansion of the present study by enhancing the number of clinical antifungal - sensitive and - resistant isolates, increase or decrease the concentrations used, or do synergistic studies on constituents. Generally, many years are required from discovery of a new antifungal to clinical use, but the development of new antifungal strategies will reduce the therapeutic time and/or increase the quality of life of patients.

\section{Literature Cited}

Ali, I., F.G. Khan, K.A. Suri, B.D. Gupta, N.K. Satti, P. Dutt, F. Afrin, G.N. Qazi, and I.A. Khan. 2010. In vitro antifungal activity of hydroxychavicol isolated from Piper betle $L$. Annals of Clinical Microbiology and Antimicrobials, 9:7.

Atkinson, R.G. 2018. Phenylpropenes: Occurrence, Distribution, and Biosynthesis in Fruit. Journal of Agricultural and Food Chemistry, 66 (10) 2259-2272.

Baron, M.D. 1996 (fourth edition). Medical Mycology. Galveston, Texas: University of Texas Galveston. pp 915916.

Bassiri-Jahromi, S., A.A. Khaksari. 2009. Epidemiological survey of dermatophytosis in Tehran, Iran from 2000 to 2005. Indian Journal of Dermatology, Venereology and Leprology. 75:142-7.

Caburian, A.B. and M.O. Osi. 2010. Characterization and Evaluation of Antimicrobial Activity of the Essential Oil from the Leaves of Piper betle L. E-International Scientific Research Journal. Vol 2 Issue 1

de Oliveira, P.F., J.M. Mendes, L.E. de Oliveira. 2013. Investigation on mechanism of antifungal activity of eugenol against Trichophyton rubrum. Medical Mycology, 51 (5) 507-13. doi: 10.3109/13693786.2012.742966.

Duke, J.A. 1985. Handbook of medicinal herbs. Boca Raton, Fla: CRC Press, Inc.

Global Action Fund for Fungal Infections (GAFFI). Accessed on December 27 2018: https://www.gaffi.org/wp-content/ uploads/GAFFI-Final-Brochure-Aug-2017-pages.pdf

Handog, E.B. and J.F. Dayrit. 2005. Mycology in the
Philippines, Revisited. Japanese Journal of Medical Mycology. 46:71-76

Maetens JA. 2004. History of the development of azole derivatives. Clin. Microbiol. Infect. 10(Suppl 1):1-10.

Moghimipour, E., A. Ameri, A. Saudatzadeh, A. Salimi, A. Siahpoosh. 2009. Formulation of an anti-dermatophyte cream from hydro-alcoholic extract of Eucalyptus camaldulensis leaves. Jundishapur Journal of Natural Pharmaceutical Products, 4(1): 32-40

Murray, P.R. 1999. Manual of Clinical Microbiology. Washington, D.C.: ASM Press

Ontengco, D.C., M. Talaue, L.J. Cruz, T.V. Capal, L.A. Dayap. 1999. The MICs of betel oil against common clinical pathogens. Acta Manilana, 47: 61-6.

Ontengco, D.C., TV Capal, C.L. Melo and L.A. Dayap. 2004. Food flavors as potential preservative systems. Acta Manilana, 52:23-27

Park, M.J., K.S. Gwak, I. Yang, W.S. Choi, H.J. Jo, J.W. Chang, E.B. Jeung, and I.G. Choi. 2007. Antifungal activities of the essential oils of Syzigium aromaticum (L.) Merr. Et Perry and Leptospermum petersonii Bailey and their constituents against various dermatophytes. Journal of Microbiology, 45 (5) 460-465

Reiss, E. and A. DiSalvo. 2018. Superficial Mycoses In: Mycology and Immunology On Line. University of South Carolina School of Medicine. Accessed on December 27, 2018: http://www.microbiologybook.org/ mycology/2018mycology-4.htm

Rimando, A.M., B. Hoon Han, J.H. Park, and M. Cantoria. 1986. Studies on the constituents of Philippine Piper betle leaves. Archives of Pharmacal Research, 9 (2). 93-97

Thomson, W.A.R. (ed.). 1978. Medicines from the Earth. Maidenhead, United Kingdom: McGraw-Hill Book Co.

Thormar, H., K.A. Hammer, C.F. Carson. 2010. Antibacterial and Antifungal Activities of Essential Oils. In: Thormar, H. (ed.). Lipids and Essential Oils as Antimicrobial Agents, pp 255-266.

Umali-Stuart, G. n.d. Ikmo. StuartXchange. Accessed on June 26, 2015: http://www.stuartxchange.org/Ikmo.html

World Health Organization. 2005. Epidemiology and management of common skin diseases in children in developing countries. Department of Child and Adolescent Health and Development. p 54 